\title{
Guidelines for the management of patients who are taking oral anticoagulants and who require dental surgery
}

\author{
Feldens CA, Vítolo MR, Drachler ML. \\ A randomized trial of the effectiveness of home visits in preventing \\ early childhood caries. Community Dent Oral Epidemiol 2007; \\ 35:215-223.
}

Scope and purpose This aims to provide healthcare professionals, including primary care dental practitioners, with clear guidance on the management of patients who are taking oral anticoagulants and who need dental surgery.

Methods Medline and Embase databases were searched from 19502006 using the following keywords: dental, surgery, oral and anticoagulants. The guideline was developed according to the guidelines of the British Committee for Standards in Haematology (BCSH), which can be seen in detail at www.bcshguidelines.com/process1.asp. The system for classification of evidence and grading of recommendations is shown in Table 1.

Review and Updating $\mathrm{BSCH}$ indicate that updating review of the guideline is planned for 2011 .

Recommendations The guideline make 5 key recommendations which are shown in table 2 .

Research recommendations None were given.

\section{Commentary}

These guidelines were developed by the BCSH and, in addition to being published in the British Dental Journal, ${ }^{1}$ are available on the web (www.bcshguidelines.com/pdf/WarfarinandOralSurgery26407.pdf). The number of people living in the community who take anticoagulants is on the rise and, as dental extraction is the most common surgical procedure, the need for clear guidance on managing these individuals is important. Indeed, as the guideline points out, there has been a good deal of historical controversy over how best to manage them.

The recommendations are based upon the balance of risks between continuing anticoagulation and postoperative haemorrhage and thrombosis. These are clearly in favour of continuing anticoagulation: there is a $1 \%$ risk of serious embolic complications including death but only a $0.2 \%$ risk of major bleeding for patients with a therapeutic INR $<4$. The dental practice procedures which require measurement of the INR are shown below:

- Endodontics (root canal treatment)

- Local anaesthesia (infiltrations, inferior alveolar nerve block, mandibular blocks)

- Extractions (single and multiple)

- Minor oral surgery

- Periodontal surgery

- Biopsies

- Subgingival scaling

The guidance also recommends two particular groups of patients who should not have surgical dental procedures in the dental practice setting, namely:

- People taking oral anticoagulants who also have co-existing medical problems, eg, liver disease, renal disease, thrombocytopaenia or who are taking antiplatelet drugs.

Such patients may have an increased risk of bleeding.

- Patients requiring surgical procedures not listed above. Such patients should be referred to a dental hospital or hospital-based oral and maxillofacial surgery department.

Although not described in the guideline or the $B D J$ publication, ${ }^{1}$ the $\mathrm{BCSH}$ does have a clear process for the development of guidelines and these are laid out on their website (see above). The process appears to fall short of the AGREE (Appraisal of Guidelines for Research and Evaluation; www.agreecollaboration.org) standards, however, as there is a lack of detail in the document or the website regarding various elements in the process. There is neither clear indication of the criteria for inclusion of the articles nor how they were appraised. Despite the guideline being authored by three people and being approved by the $\mathrm{BCSH}$, there is no indication of the contribution of potential stakeholders (general dental practitioners, oral surgeons, general medical practitioners, patients, haematologists, etc). The guideline was circulated to a large group for comment prior to 


\begin{tabular}{|c|c|}
\hline Evidence level & Required standard \\
\hline la & Evidence obtained from meta-analysis of RCT \\
\hline $\mathrm{lb}$ & Evidence obtained from at least one RCT \\
\hline Ila & Evidence obtained from at least one well-designed controlled study without randomisation \\
\hline $\mathrm{llb}$ & Evidence obtained from at least one other type of well-designed quasi-experimental study* \\
\hline III & Evidence obtained from well-designed nonexperimental descriptive studies, eg, comparative studies, correlation studies and case studies \\
\hline IV & Evidence obtained from expert committee reports or opinions and/ or clinical experiences of respected authorities \\
\hline \multicolumn{2}{|c|}{ Grades of recommendations } \\
\hline A & $\begin{array}{l}\text { Requires at least one RCT as part of a body of literature of overall good quality and consistency addressing specific recommendation } \\
\text { (evidence levels la, lb) }\end{array}$ \\
\hline B & Requires availability of well-conducted clinical studies but no RCT on topic of recommendation (evidence levels Ila, IIb, III) \\
\hline C & $\begin{array}{l}\text { Requires evidence obtained from expert committee reports or opinions and/ or clinical experiences of respected authorities. } \\
\text { Indicates an absence of directly applicable clinical studies of good quality (evidence level IV) }\end{array}$ \\
\hline
\end{tabular}

\section{Table 2. Key recommendations}

\section{Recommendation}

Grade of recommendation and evidence level

Risk of significant bleeding in patients taking oral anticoagulants who have stable INR in the therapeutic range 2-4 $(i e,<4)$ is very small and the risk of thrombosis may be increased if oral anticoagulants are temporarily discontinued. Oral anticoagulants should not be discontinued in the majority of patients requiring outpatient dental surgery including dental extraction

For patients stably anticoagulated on warfarin (INR, 2-4) and who are prescribed a single dose of antibiotics as prophylaxis against endocarditis, there is no need to alter their anticoagulant regimen

Grade A, level Ib

The risk of bleeding may be minimised by:

a. Use of oxidised cellulose (Surgicel, Johnson \& Johnson Medical, New Brunswick, NJ, USA or collagen sponges and sutures

b. Using $5 \%$ tranexamic acid* mouthwashes used four times/ day for 2 days

For patients who are stably anticoagulated on warfarin, a check INR is recommended $72 \mathrm{~h}$ before dental surgery

Patients taking warfarin should not be prescribed nonselective NSAID and COX-2 inhibitors as analgesia following dental surgery

*Tranexamic acid is not readily available in most primary care dental practices.

INR, International normalised ratio; NSAID, nonsteroidal anti-inflammatory drugs; COX-2, cyclo-oxygenase 2 (subtype of prostaglandin-endoperoxide synthase that plays an important role in many cellular processes and inflammation).

final publication but no information is given about the comments or changes resulting from this.

Many of these process issues may have been carried out but, because they are not dealt with in the documentation or on the website, uncertainty results. Some will say that including these additional details is unnecessary when what is needed is clear, unequivocal recommendations - which this guidance delivers - but providing the additional detail does better enable the reader to judge the quality of the development process. Well-funded national guidance development groups such as NICE (National Institute of Health and Clinical Excellence) and SIGN (Scottish Intercollegiate Guidelines Network) do this and the quality of their products is recognised.

It worth noting that the imminent NICE guidance, currently in the consultation draft (www.nice.org.uk/guidance/index.jsp?action= download\&o=38152), recommends, "Antibiotic prophylaxis against infective endocarditis (IE) is not recommended for patients at risk of IE undergoing dental procedures": the second recommendation of the BCSH will become superfluous if, as anticipated, the NICE recommendation is not amended.

That said, the guidance is relatively straightforward and highlights the key issues when managing patients taking anticoagulants. It also accords with what is taught in most dental schools and what, in discussion with general practitioner colleagues, they say they currently practice. For those wanting more detailed background information and practical information, I recommend looking at the North West Medicines Information Centre document which is available on the web (www.ukmi.nhs.uk/med_info/documents/Dental_Patient_on_ Warfarin.pdf) which was last revised in March 2007.

\section{Derek Richards}

\section{Centre for Evidence-based Dentistry, Oxford, UK}

1. Perry DJ, Noakes TJ, Helliwell PS. Guidelines for the management of patients on oral anticoagulants requiring dental surgery. Br Dent J 2007; 203:389-393.

Evidence-Based Dentistry (2008) 9, 5-3. doi:10.1038/sj.ebd.6400556 